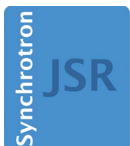

JOURNAL OF SYNCHROTRON RADIATION

ISSN 1600-5775

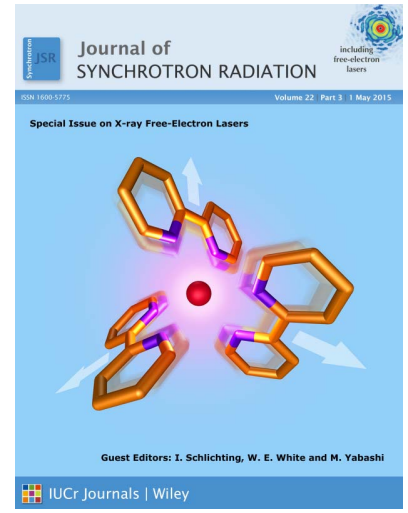

\section{An introduction to the special issue on $X$-ray free-electron lasers}

\author{
Ilme Schlichting, ${ }^{\text {a* }}$ William E. White ${ }^{\mathrm{b} *}$ and Makina Yabashi ${ }^{\mathrm{c} *}$
}

aDepartment of Biomolecular Mechanisms, Max Planck Institute for Medical Research, Jahnstrasse 29, 69120 Heidelberg, Germany, 'binac Coherent Light Source, SLAC National Accelerator Laboratory, 2575 Sand Hill Road, Menlo Park, CA 94025, USA, and ${ }^{\mathbf{c}}$ RIKEN SPring-8 Center, Kouto 1-1-1, Sayo, Hyogo 679-5148, Japan. *Correspondence e-mail: jsr@mpimf-heidelberg.mpg.de, wewhite@slac.stanford.edu, yabashi@spring8.or.jp

This issue of the Journal of Synchrotron Radiation is a special issue on X-ray free-electron lasers. Here, a brief introduction to these special issue papers is given.

X-ray free-electron lasers (FELs) are coming of age. 15 years ago, first lasing was achieved with a SASE (self-amplified spontaneous emission) FEL at the Tesla Test Facility at DESY, the forerunner of the VUV FEL FLASH in Hamburg. Hard X-rays became available a little over five years ago when the Linac Coherent Light Source (LCLS) at SLAC National Accelerator Laboratory opened its doors to users, followed by the SPring-8 Angstrom Compact Free Electron Laser (SACLA) three years ago. With their femtosecond short pulses and ultra-high peak brilliance, FELs have enabled the advance of many different areas of science. To name just a few applications, FELs have provided novel insight into light-matter interactions, enabled ultrafast timeresolved measurements at high spatial resolution, provided a tool long-awaited for the study of matter under extreme conditions and of nanoscale objects, including biological materials.

It thus seems timely for a special FEL-related issue in the Journal of Synchrotron Radiation (JSR). Feature articles describe the sources and facilities: LCLS, SACLA and the VUV FEL FERMI, giving insight in the development of and possibilities at these facilities. Beamline papers describe the instruments, providing detailed information on the experimental capabilities along with recent scientific results highlighting the possibilities. Various laser systems for time-resolved measurements are described, giving background information on planning experiments, along with contributions on X-ray detectors, non-linear signal response issues and data acquisition systems. Diagnostics are critical to single-pulse machines like FELs. Thus, some contributions describe diagnostic tools and techniques, including new approaches for focus characterization driven by developments in nanofocusing to study smaller and smaller objects at increasingly higher flux densities. New capabilities are constantly being developed at FEL sources and a few such capabilities are described, including spectroscopy techniques and the use of the seeded beam in crystallography. The increasing interest in using these machines is not only accommodated by developments in multiplexing the single FEL beam to different instruments or to use the spent beam but also by the construction of a number of new FELs such as FLASH2 and the European XFEL in Germany, the SwissFEL in Switzerland, and the PAL-XFEL in Korea. A future special issue on FELs in JSR will focus on these upcoming facilities. 